\title{
Neuromyelitis Optica Spectrum Disorders Associated To Posterior Reversible Encephalopathy Syndrome: Pathogenesis, Diagnosis, Treatment and Evolution: A Case Report and Literature Review
}

\author{
Benmoh $\mathbf{Y}^{1 *}$, Hamid $\mathbf{M}^{1}$, Arrami $\mathrm{A}^{2}$, Alaoui $\mathrm{MY}^{3}$, Satte $\mathrm{A}^{4}$, Mounach $\mathrm{J}^{5}$, Bichra $\mathrm{MZ}^{3}$, Elfenni $\mathrm{J}^{2}$ and Bourazza $\mathrm{A}^{1}$ \\ ${ }^{1}$ Department of Neurology, Mohamed V Military Teaching Hospital, Mohamed V University, Rabat, Morocco \\ ${ }^{2}$ Department of Radiology, Mohamed V Military Teaching Hospital, Mohamed V University, Rabat, Morocco \\ ${ }^{3}$ Department of Psychiatry, Mohamed V Military Teaching Hospital, Mohamed V University, Rabat, Morocco \\ ${ }^{4}$ Department of Neurophysiology, Mohamed V University, Morocco \\ ${ }^{5}$ Department of Neurophysiology, Sidi Mohamed Ben Abdellah University, Morocco
}

\begin{abstract}
Introduction: Neurmyelitis Optica Spectrum Disorders (NMOSD) is a rare central nervous demyelinating autoimmune disease. Posterior Reversible Encephalopathy Syndrome (PRES) is a reversible subcortical vasogenic brain edema with acute neurological symptoms and specific radiologic findings. We report a rare case associating both NMOSD and PRES as revealing form.

Case Report: A 50 years old woman, with previous history of 3 neurologic episodes made of visual loss, lower limb weakness and paraethesia, regressing spontaneously in the past 3 years. 7 days before her admission, she presented rapidly progressive weakness of both lower limbs, followed by urinary retention, headache and fluctuant awareness. Clinical examination found symmetric flaccid paraparesis, with sensory level at D6. Spinal cord MRI showed thoracic longitudinally extensive transverse myelitis compatible with NMOSD pattern. Cerebral MRI showed increased signal intensity in T2 and FLAIR weighted images localized in: subcortical dominant in parieto-occipital regions and holohemispheric watershed pattern compatible with PRES. Cerebrospinal fluid study objectified pleocytosis to neutrophils, and Visual evocked potential study found bilateral demyelinating optic neuritis. The Aquaporin4-specific IgG was positive, with large negative paraclinical tests eliminating alternative etiologies or association. The fail of initial bolus methyprednisolone, leaded to a cure of human immunoglobulin and cyclophosphamide with favorable course. Cerebral MRI control was performed 3 months and 12 months later, and showed complete regression of signal abnormalities. Spinal cord MRI control showed thoracic spine atrophy.
\end{abstract}

Conclusion: Severe impairment in blood brain barrier water channels by aquaporin4-specific lgG cause a knockout phenotype with an increase of brain edema. This suggests that NMOSD acute attack and PRES may co-occur like in our case. Few cases are reported over the literature, for this association, especially as a revealing form. When the precipitating cause is treated or eliminated, PRES lesions are usually reversible. Thus, in PRES associated to NMOSD, the treatment refers to immunotherapy.

Keywords: Neuromyelitis Optica Spectrum Disorders (NMOSD); Posterior Reversible Encephalopathy Syndrome (PRES); Aquaporin channelopathy; Aquaporin 4-specific IgG; Longitudinally Extensive Transverse Myelitis (LETM); Optic Neuritis (ON); Blood Brain Barrier (BBB); Multiple Sclerosis (MS)

Abbreviations: AQP4: Aquapor in-4; lgG: Immunoglobulin G; LETM: Longitudinally Extensive Transverse Myelitis lesions; NMOSD: Neuromyelitis Optica Spectrum Disorders.

\section{Introduction}

Neurmyelitis optica is a rare central nervous demyelinating autoimmune disease, initially known as "Devic's Disease" with recurrent optic nerve attacks and longitudinally extensive transverse myelitis. The identification of anti-aquaporin 4 antibody leaded to an increased understanding of its pathogenesis. This resulted into wider spectrum of clinical manifestation and neuroimaging features, with the revision of more specific diagnostic criteria of larger pathology: Neuromyelitis Optica Spectrum Disorders (NMOSD) [1,2]. On the other hand, Posterior Reversible Encephalopathy Syndrome (PRES) is defined as a reversible subcortical vasogenic brain edema with acute neurological symptoms and specific radiologic findings [3-5]. We report a rare case associating both NMOSD and PRES as revealing form, with literature review of possible commune pathogenesis, their specific clinical and radiological findings, and the course after therapy.

\section{Case Report}

A 50 years old woman, with previous history of osteoporosis, was admitted for rapidly progressive weakness of both lower limbs. Three years before her admission, she presented the first neurologic episode made of left visual loss with right lower limb weakness, regressing spontaneously one month later. Thirteen months later, she presented two other neurologic episodes made of paraesthesia and stringing in both lower limbs, regressing spontaneously. The recent episode was evolving seven days before the admission, made of rapidly progressive weakness of both lower limbs, associated to paraesthesia. Six days later, the evolution was complicated

*Corresponding author: Benmoh Y, Department of Neurology, Mohamed V Military Teaching Hospital, Mohamed V University, Rabat, Morocco, Tel: +212675619652; E-mail: youssouf1680@gmail.com

Received October 31, 2018; Accepted November 19, 2018; Published November 24, 2018

Citation: Benmoh Y, Hamid M, Arrami A, Alaoui MY, Satte A, et al. (2018) Neuromyelitis Optica Spectrum Disorders Associated To Posterior Reversible Encephalopathy Syndrome: Pathogenesis, Diagnosis, Treatment and Evolution: A Case Report and Literature Review. J Neurol Neurophysiol 9: 472. doi:10.4172/2155-9562.1000472

Copyright: @ 2018 Benmoh Y, et al. This is an open-access article distributed under the terms of the Creative Commons Attribution License, which permits unrestricted use, distribution, and reproduction in any medium, provided the original author and source are credited. 
Citation: Benmoh Y, Hamid M, Arrami A, Alaoui MY, Satte A, et al. (2018) Neuromyelitis Optica Spectrum Disorders Associated To Posterior Reversible Encephalopathy Syndrome: Pathogenesis, Diagnosis, Treatment and Evolution: A Case Report and Literature Review. J Neurol Neurophysiol 9: 472. doi:10.4172/2155-9562.1000472

by acute urinary retention associated to fluctuating awareness and moderate diffuse headache. There were no extra-neurologic functional symptoms (respiratory, digestive, cardiac, rheumatologic or fever). General examination found conscious patient, and stable hemodynamic and respiratory parameters (no arterial hypertension). The neck was supple. The neurologic examination found symmetric flaccid paraparesis quoted to $2 / 5$, with sensory level at D6. Thus the clinical presentation was acute encephalomyelopathy in 50 years old woman. Spinal cord MRI were performed and showed increased signal intensity in T2 and Flair weighted images. The signal was extended longitudinally to more than 5 vertebral bodies, and transversally limited in central area of the spine (Figure 1). Cerebral MRI showed increased signal intensity in T2 and FLAIR weighted images localized in: subcortical, periventricular, internal capsule, thalamus, lenticular nucleus, caudate nucleus and area postrema (Figure 2). The cerebrospinal fluid study objectified pleocytosis (9 white cells/ $\mathrm{mm}^{3}$ made of neutrophils), with elevated protein $(0.47 \mathrm{~g} / \mathrm{L})$. There was no intrathecal IgG synthesis as much as no oligoclonal bands. Visual acuity was reduced to $8 / 10$ in both eyes with altitudinal hemianopsia in visual field study. Visual Evocked Potential study (VEP) found bilateral increased P100 wave latency secondary to bilateral demyelinating optic neuropathy.

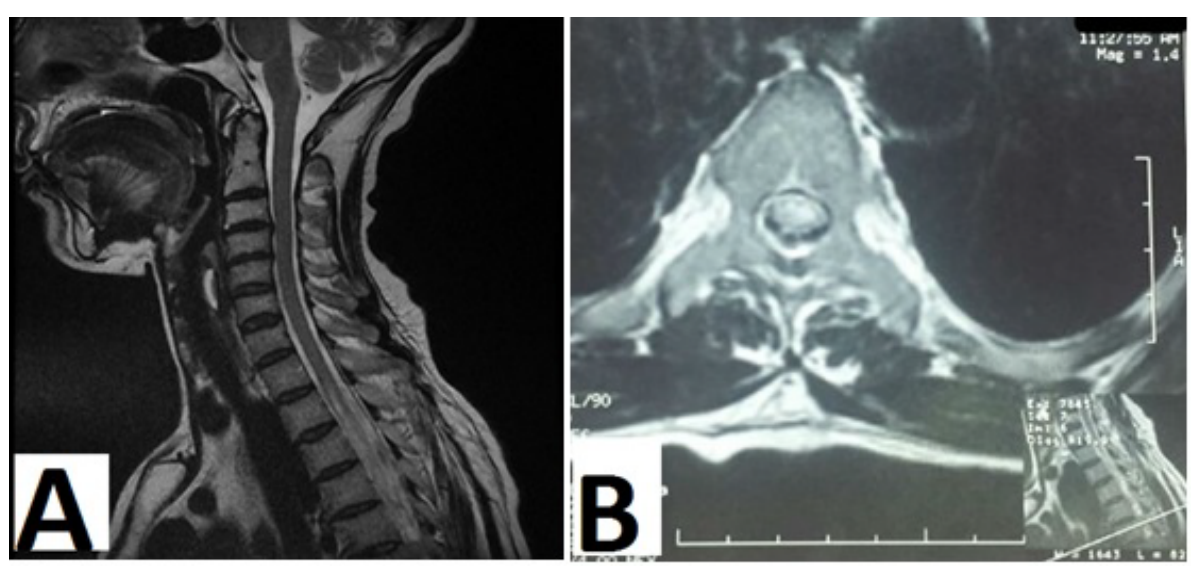

Figure 1: Spinal cord MRI in sagittal (A) and axial (B) T2 weighted images showing dorsal spine enlargement with increased signal intensity extensive longitudinally to more than 5 vertebral bodies in sagittal section (A), and transversally to more than $50 \%$ of spine area, dominant in central zone (B). This aspect is defined as Longitudinally Extensive Transverse Myelitis (LETM), a specific pattern of NMOSD.

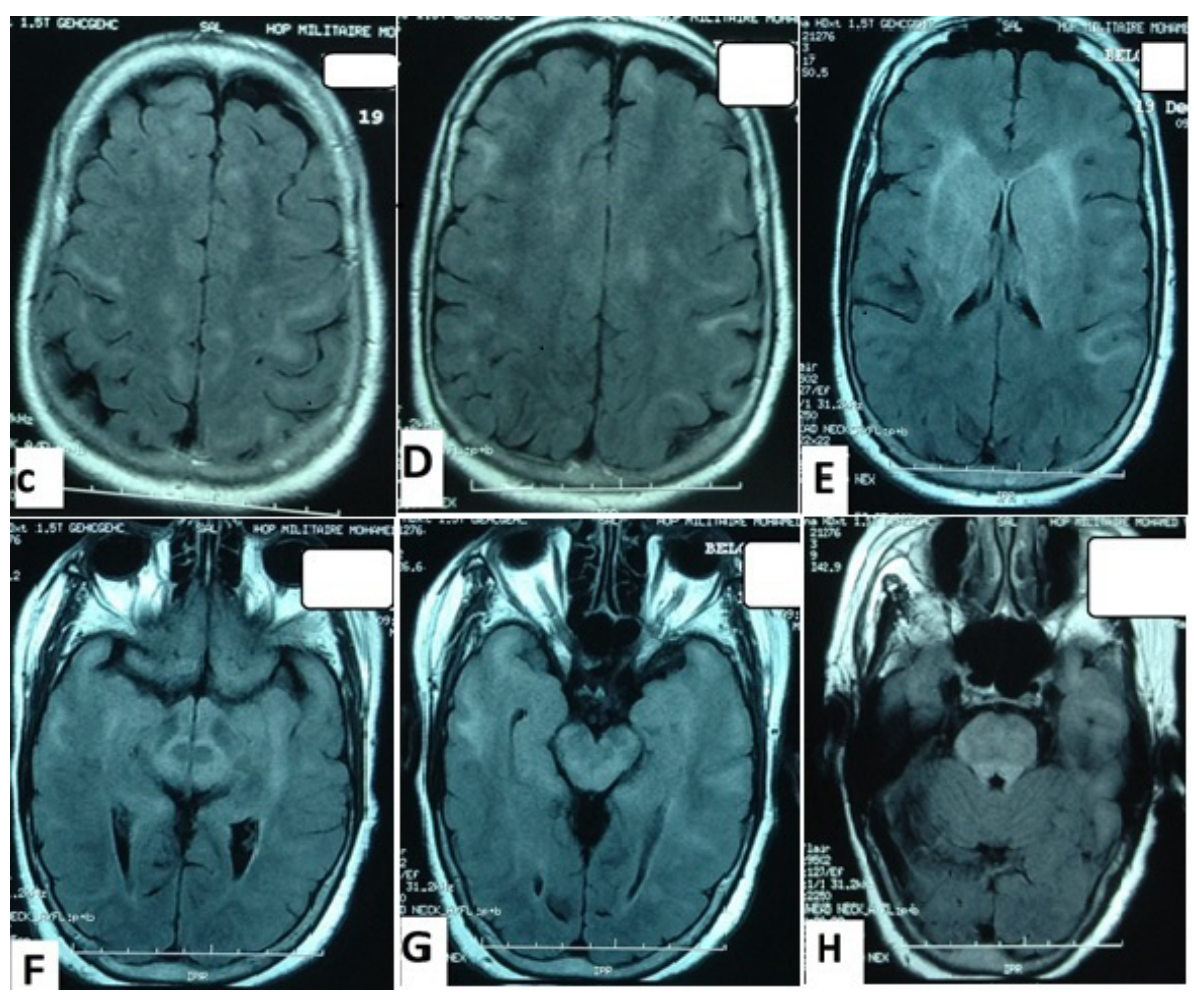

Figure 2: Cerebral MRI in axial FLAIR weighted images, showing increased signal intensity with the dominant asymetric parieto-occipital pattern and holo-hemispheric watershed pattern compatible with PRES (C,D); parenchyma increased signal intensity around lateral, third and fourth ventricle $(E, F, G, H)$ including thalamus, internal capsule, caudate and lenticular nucleus and area postrema, a pattern compatible with NMOSD 
Citation: Benmoh Y, Hamid M, Arrami A, Alaoui MY, Satte A, et al. (2018) Neuromyelitis Optica Spectrum Disorders Associated To Posterior Reversible Encephalopathy Syndrome: Pathogenesis, Diagnosis, Treatment and Evolution: A Case Report and Literature Review. J Neurol Neurophysiol 9: 472. doi:10.4172/2155-9562.1000472

Large para-clinical tests were performed according to the diagnosis of acute encephalomyelitis with bilateral optic demyelinating neuropathy. The complete count blood was normal, as much as the $\mathrm{C}$ reactive protein and erythrocyte sedimentation rate. B12, folic acid, phosphocalcic, angiotensin converting enzyme and cooper levels were normal. Multiple infectious serologies were realized and went all negative (tuberculosis, syphilis, borreliosis, brucellosis, VIH, hepatitis B and C, herpes simplex, toxoplasmosis). Anti-DNA and anti-nuclear antibody were negative. The salivary gland biopsy went normal. Anti-AQP4 antibody was tested by cell based assay and went positive. Thus, the patient fulfills enough criteria of the international consensus diagnostic criteria for neuromyelitis optica spectrum disorders (acute myelitis with positive AQP4-specific IgG). Also radiologic finding meet enough characteristic for PRES: asymmetric edema dominating in parieto-occipital regions with holo-hemispheric watershed pattern. The patient was treated by $3 \mathrm{~g}$ bolus methylprednisolone ( $1 \mathrm{~g}$ daily), followed by oral prednisone at $1 \mathrm{mg} / \mathrm{kg} / \mathrm{d}$. the neurologic state keep worsening with aggravation of paraparesis associated to vomiting and nausea. The team decision was to start human immunoglobulin at the dose of $0.4 \mathrm{~g} / \mathrm{Kg} / \mathrm{d}$ for five days. The evolution was favorable with complete regression of paraparesis, headache, vomiting and sphincter disorders. Patient vigilance went normal. Regarding maintenance treatment, the team decided to administer cyclophosphamid $\left(600 \mathrm{mg} / \mathrm{m}^{2} / 2\right.$ months $)$ associated to oral prednisone $(1 \mathrm{mg} / \mathrm{Kg} / \mathrm{d}$ for 6 weeks than progressive digression to $10 \mathrm{mg} / \mathrm{d}$ ). Cerebral MRI control was performed 3 months and 12 months later, and showed complete regression of signal abnormalities (Figure 3). Spinal cord MRI control showed cervical spine atrophy.

\section{Discussion}

NMOSD incidence varies from 0.053 to 0.4 per 100,000 people [6], and the prevalence from 0.3 to 4.4 per 100,000 . Its sex-ratio $\mathrm{F} / \mathrm{M}$ is above $3: 1$ to $9: 1$. The median age is 39 years, and asian and African-american population are disproportionally affected [6,7]. The

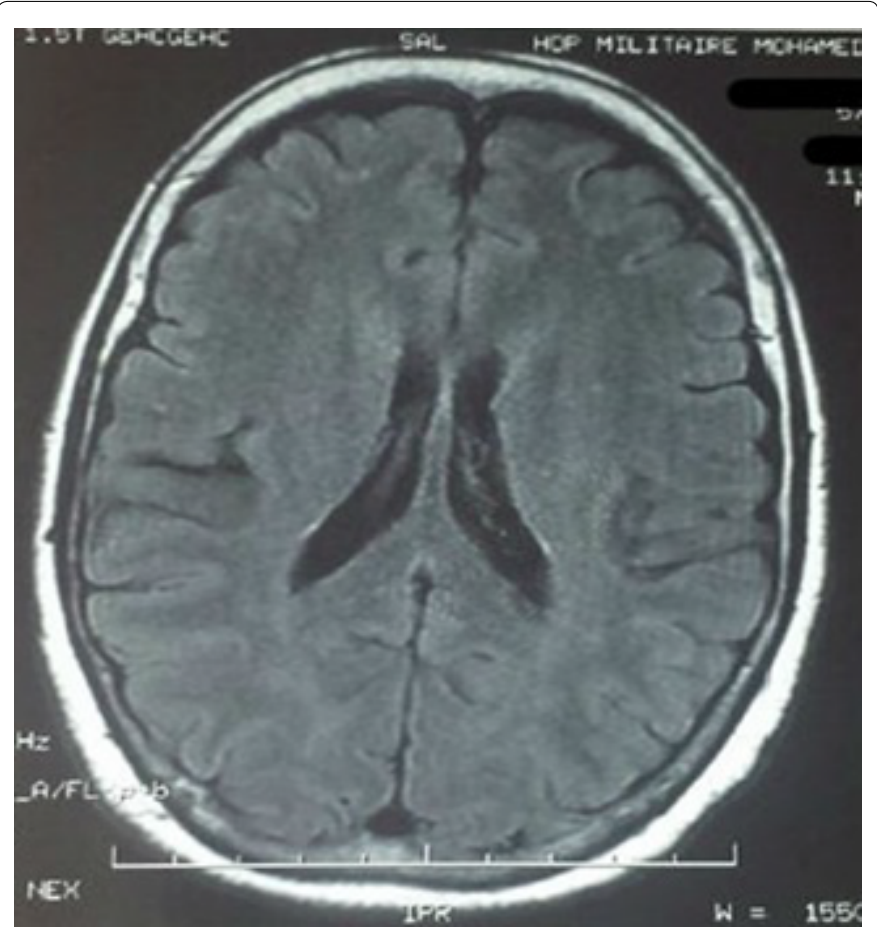

Figure 3: Cerebral MRI control in axial FLAIR weighted image showing complete regression of increased signal intensity 12 months later. previous lacks of distinguishing biomarker, made NMOSD historically misdiagnosed as multiple sclerosis. There are several clinical symptoms suggesting NMOSD: uni/bilateral optic neuritis, altitudinal hemianopsia, narcolepsy, insomnia, endocrinopathies, area postrema syndrome (nausea, vomiting and hiccup), symmetric paraparesis, tonic painful spasm, neuropathic pruritus $[8,9]$. In $85 \%$ of NMOSD cases, patients had a relapsing course, while only $15 \%$ of cases had a monophasic illness (Devic's disease) [8,9]. Spine, cerebral and optic nerve neuroimaging had special findings distinguished from multiple sclerosis (MS) ones. Spine lesions may be cervical or thoracic, more central and longitudinally extensive to more than 3 vertebral bodies [8]. Optic nerve MRI abnormalities are more posterior with enhancement of the chiasma [10,11]. Cerebral MRI typical aspect are localized in the astroglial foot processing in subpial and subependymal zones around lateral, third and fourth ventricles. These localizations follow the AQP4enriched regions [12]. In contrast with MS, CSF study in NMOSD show pleocytosis more to neutrophils and eosinophils, with elevated protein and infrequently oligoclonal immunoglobulin bands [13]. Recently, the discovery of aquaporin antibody increased the pathogenesis understanding, and leaded to establish new NMOSD diagnostic criteria (Table 1) [2]. In the presence of AQP4-IgG, the presence of one clinical

\section{Diagnostic criteria for NMOSD with AQP4-IgG}

1. At least 1 core clinical characteristic.

2. Positive test for AQP4-IgG using best available detection method (cell-based assay strongly recommended).

\section{Exclusion of alternative diagnoses.}

Diagnostic criteria for NMOSD without AQP4-IgG or NMOSD with unknown AQP4-IgG status

1. At least 2 core clinical characteristics occurring as a result of one or more clinical attacks and meeting all of the following requirements:

a. At least 1 core clinical characteristic must be optic neuritis, acute myelitis with LETM, or area postrema syndrome.

b. Dissemination in space (2 or more different core clinical characteristics).

c. Fulfillment of additional MRI requirements, as applicable.

2. Negative tests for AQP4-IgG using best available detection method, or testing unavailable.

3. Exclusion of alternative diagnoses.

\section{Core clinical characteristics}

1. Optic neuritis

2. Acute myelitis

3. Area postrema syndrome: episode of otherwise unexplained hiccups or nausea and vomiting

4. Acute brainstem syndrome

5. Symptomatic narcolepsy or acute diencephalic clinical syndrome with NMOSD-typical diencephalic MRI lesions.

6. Symptomatic cerebral syndrome with NMOSD-typical brain lesions. Additional MRI requirements for NMOSD without AQP4-IgG and NMOSD with unknown AQP4-IgG status

1. Acute optic neuritis: requires brain MRI showing (a) normal findings or only nonspecific white matter lesions, OR (b) optic nerve MRI with T2-hyperintense lesion or T-weighted gadolinium enhancing lesion extending over $>1 / 2$ optic nerve length or involving optic chiasm.

2. Acute myelitis: requires associated intramedullary MRI lesion extending over $>3$ contiguous.

Segments (LETM) OR $>3$ contiguous segments of focal spinal cord atrophy in patients with history compatible with acute myelitis.

3. Area postrema syndrome: requires associated dorsal medulla/area postrema lesions.

4. Acute brainstem syndrome: requires associated periependymal brainstem lesions.

Table 1: NMOSD diagnostic criteria in adult patients according to the Internationa Panel for Neuromyelitis optica Diagnosis IPND 2015 [2]. 
Citation: Benmoh Y, Hamid M, Arrami A, Alaoui MY, Satte A, et al. (2018) Neuromyelitis Optica Spectrum Disorders Associated To Posterior Reversible Encephalopathy Syndrome: Pathogenesis, Diagnosis, Treatment and Evolution: A Case Report and Literature Review. J Neurol Neurophysiol 9: 472. doi:10.4172/2155-9562.1000472

core with elimination of other differential diagnosis, are sufficient to confirm the diagnosis. This was the case of our patient, who presented Longitudinally Extensive Transverse Myelitis (LETM) with positive AQP4-specific IgG, after elimination of alternative infectious and systemic diseases.

PRES was first described two decades ago. Its pathogenesis is not fully understood, but endothelial impairment and hyper perfusion are the key factors. PRES occur in the setting of renal failure, blood pressure fluctuation, auto-immune disorders, eclampsia or cytotoxic drugs. These situations cause reversible subcortical vasogenic brain edema, manifesting by acute neurological symptoms (encephalopathy, seizure or status epilepticus, headache, visual disturbance, focal deficit) [14]. Brain imaging confirms the PRES diagnosis, even if no diagnosis gold standard exists. In $70 \%$ of cases, three MRI variations exist: dominant parieto-occipital pattern, holo-hemispheric watershed pattern, superior frontal sulcus pattern and [15]. Our patient presented impaired awareness with moderate diffuse headache, associated to the first two MRI patterns, confirming the coexistence of NMOSD and PRES.

Concerning NMOSD pathogenesis, the exact causes stay unknown. Nevertheless, antibody-mediated disorders targeting astrocyte are widely recognized $[16,17]$. Aquaporin are a group of water channel regulating water transport and present in many organs (kidney, secretatory glands, gastrointestinal tract, nervous system and muscle). In central nervous system, aquaporins 4 (main target in NMOSD) are concentrated in the Blood Brain Barrier (BBB) included in the astrocyt foot process membrane. In NMOSD, the primary inflammation site is the perivascular space where localized the AQP4 immunoreactivity. The AQP4 surface expression is down-regulated by selective IgG bindings AQP4, leading to higher BBB permeability and complement activation with subsequent tissue infiltration of leucocytes, lymphocyte and NK cells. The result is an astrocytopathy with secondary oligodendrocyte and neuron involvement [17-20]. Severe impairment in BBB water channels by aquaporin4-specific IgG cause a knock-out phenotype with an increase of brain edema. This suggests that NMOSD and PRES may co-occur like in our case. Very few cases are reported over the literature with this association, especially as a revealing form [21].

No specific treatment exists for PRES. When the precipitating cause is treated or eliminated, PRES lesions are usually reversible. Thus, in PRES associated to NMOSD, the treatment refers to immunotherapy. The acute attack could be treated by high dose corticosteroids $\left(1^{\text {st }}\right.$ line), plasmapheresis or human immunoglobulin ( $2^{\text {nd }}$ line) $[22,23]$. Unlike MS, NMOSD did not respond to immunomodulatory therapies which may be detrimental. The maintenance treatment is primordial to reduce number and severity of relapses. It is based on immunosuppressive therapies, most commonly: Rituximab, Mycophenolate mofetil, azathioprine, but also methotrexate and cyclophosphamide [24]. In our patient, we choose human immunoglobulin as second line therapy for the acute attack and cyclophosphamide as maintenance therapy with favorable clinical and radiographic outcome.

\section{Conclusion}

We learn from this case that NMOSD and PRES may co-exist. This suggests commune pathogeny where AQP4-IgG lead to higher BBB permeability and brain edema. The association should be suspected in patient presenting suggesting PRES symptoms: encephalopathy, seizure or status epilepticus, headache, visual disturbance, focal deficit. Cerebral MRI confirms PRES pattern and their reversibility. Prognosis depends on timeliness NMOSD treatment for the acute attack, but also the maintenance treatment.

\section{References}

1. Wingerchuk DM, Lennon VA, Pittock SJ, Lucchinetti CF, Weinshenker BG (2006) Revised diagnostic criteria for neuromyelitis optica. Neurology 66: 14851489.

2. Fujihara B, Greenberg A, Jacob S, Jarius M, Lana-Peixoto M, et al. (2015) International consensus diagnostic criteria for neuromyelitis optica spectrum disorders. Neurology 85: 177-89.

3. Hauser RA, Lacey DM, Knight MR (1988) Hypertensive encephalopathy: Magnetic resonance imaging demonstration of reversible cortical and white matter lesions. Arch Neurol 45: 1078-1083.

4. Schwaighofer BW, Hesselink JR, Healy ME (1989) MR demonstration of reversible brain abnormalities in eclampsia. J Comput Assist Tomogr 13: 310-312.

5. Hinchey J, Chaves C, Appignani B (1996) A reversible posterior leukoencephalopathy syndrome. N Engl J Med 334: 494-500.

6. Marrie RA, Gryba C (2013) The incidence and prevalence of neuromyelitis optica: A systematic review. Int JMSCare 15: 113-118.

7. Quek AM, Mckeon A, Lennon VA (2012) Effects of age and sex on aquaporin-4 autoimmunity. Arch Neurol 69: 1039-1043.

8. Jarius S, Wildemann B (2012) The case of the Marquis de Causan (1804): An early account of visual loss associated with spinal cord inflammation. J Neurol 259: 1354-1357

9. Wingerchuk DM, Lennon VA, Lucchinetti CF (2007) The spectrum of neuromyelitis optica. Lancet Neurol 6: 805-815.

10. Khanna S, Sharma A, Huecker J (2012) Magnetic resonance imaging of optic neuritis in patients with neuromyelitis optica versus multiple sclerosis. J Neuroophthalmol 32: 216-220.

11. Storoni M, Davagnanaml M (2013) Distinguishing optic neuritis in neuromyelitis optica spectrum disease from multiple sclerosis: A novelmagnetic resonance imaging scoring system. J Neuroophthalmol 33: 123-127.

12. Pittock SJ, Weinshenker BG, Lucchinetti CF (2006) Neuromyelitis optica brain lesions localized at sites of high aquaporin 4 expression. Arch Neurol 63: 964-968.

13. Jarius S, Paul F, Franciotta D (2011) Cerebrospinal fluid findings in aquaporin-4 antibody positive neuromyelitis optica: Results from 211 lumbar punctures. $J$ Neurol Sci 306: 82-90

14. Li Y, Gor D, Walicki D (2012) Spectrum and potential pathogenesis of reversible posterior leukoencephalopathy syndrome. J Stroke Cerebrovasc Dis 21: 873-882.

15. Bartynski WS, Boardman JF (2007) Distinct imaging patterns and lesion distribution in posterior reversible encephalopathy syndrome. AJNR Am J Neuroradiol 28: 1320-1327.

16. Wingerchuk DM (2007) Neuromyelitis optica: New findings on pathogenesis Int Rev Neurobiol 79: 665-688.

17. Lucchinetti CF, Mandler RN, McGavern D, Bruck W, Gleich G, et al. (2002) A role for humoral mechanisms in the pathogenesis of Devic's neuromyelitis optica, Brain. J Neurol 125: 1450-1461.

18. Lucchinetti CF, Guo Y, Popescu BF, Fujihara K, Itoyama Y, et al. (2014) The pathology of an autoimmune astrocytopathy: Lessons learned from neuromyelitis optica. Brain Pathol 24: 83-97.

19. Papadopoulos MC, Verkman AS (2012) Aquaporin 4 and neuromyelitis optica Lancet Neurol 11: 535-544.

20. Amiry-Moghaddam M, Frydenlund DS, Ottersen OP (2004) Anchoring of aquaporin-4 in brain: molecular mechanisms and implications for the physiology and pathophysiology of water transport. Neuroscience 129: 999-1010.

21. Magaña SM, Matiello M, Pittock SJ (2009) Posterior reversible encephalopathy syndrome in neuromyelitis optica spectrum disorders. Neurology 72: 712-717.

22. Abboud H, PetrakA, Mealy M, Sasidharan S, Siddique L, et al. (2016) Treatment of acute relapses in neuromyelitis optica: Steroids alone versus steroids plus plasma exchange. Mult Scler 22: 185-192.

23. Bonnan M, Cabre $P$ (2012) Plasma exchange in severe attacks of neuromyelitis optica. Mult Scler Int 7: 87-630.

24. Biswas A, Mukherjee A (2015) Therapy of NMO spectrum disorders. Ann Indian Acad Neurol 18: 16-23. 